\title{
On the critical energy required for homogeneous nucleation in bubble chambers employed in dark matter searches
}

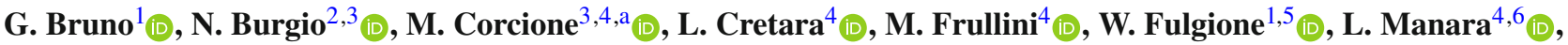 \\ A. Quintino $^{3,4}$ (D) A. Santagata ${ }^{2}$ (D) L. Zanotti ${ }^{6,7}$ \\ ${ }^{1}$ INFN, LNGS, Via G. Acitelli 22, Assergi, 67100 L’Aquila, Italy \\ ${ }^{2}$ ENEA, Centro Ricerche Casaccia, Via Anguillarese 301, 00123 S. Maria di Galeria, Rome, Italy \\ ${ }^{3}$ INFN, Sezione di Roma La Sapienza, P.le Aldo Moro 2, 00185 Rome, Italy \\ ${ }^{4}$ DIAEE, Sapienza Università di Roma, Via Eudossiana 18, 00184 Rome, Italy \\ ${ }^{5}$ INAF, Osservatorio Astrofisico di Torino, Via Osservatorio 30, 10025 Pino Torinese, Turin, Italy \\ ${ }^{6}$ INFN, Sezione di Milano-Bicocca, P.za della Scienza 3, 20126 Milan, Italy \\ ${ }^{7}$ Dip. di Fisica, Università di Milano-Bicocca, P.za della Scienza 3, 20126 Milan, Italy
}

Received: 3 January 2019 / Accepted: 15 February 2019 / Published online: 28 February 2019

(C) The Author(s) 2019

\begin{abstract}
Two equations for the calculation of the critical energy required for homogeneous nucleation in a superheated liquid, and the related critical radius of the nucleated vapour bubble, are obtained, the former by the direct application of the first law of thermodynamics, the latter by considering that the bubble formation implies the overcoming of a barrier of the free enthalpy potential. Compared with the currently used relationships, the present equations, still allowing for reversible processes only, lead to thermodynamic energy thresholds of the bubble chambers employed in dark matter searches that are closer to the experimental values.
\end{abstract}

\section{Introduction}

Bubble chambers using superheated liquids have been widely employed in high-energy physics for several decades after the invention of Glaser dated back to 1952 [1]. Recently, variants of such detectors are exploited in the search for dark matter in the form of weakly interacting massive particles (WIMPs), the main difference from the standard bubble chambers being the fact that the target liquid is continuously maintained in the metastable superheated state, instead of for just a few milliseconds [2-7].

In both applications, bubble nucleation is the result of a highly localized deposition of at least the minimum amount of energy required for the formation of a bubble of critical size, as postulated by Seitz in his "thermal spike" theory [8], which is the model currently accepted as the best explanation available for radiation-induced nucleation in super-

\footnotetext{
a e-mail: massimo.corcione@uniroma1.it
}

heated liquids. The minimum amount of energy to be released as a thermal spike to produce a bubble nucleation, typically called critical energy, is generally expressed as the sum of a number of terms, this number varying with the assumptions made by each investigator. Moreover, also the value of the critical bubble radius, which enters directly into the calculation of the critical energy, depends on the assumptions made for its evaluation. Indeed, very often the theoretical values of the critical energy, i.e., the thermodynamic energy thresholds, are lower, sometimes drastically, than the corresponding experimental values. On the other hand, the relatively low threshold needed for WIMP-recoil detection asks to be the most accurate as possible in the prediction of the critical energy required for bubble nucleation, which also helps to provide a correct explanation for why the calibration results give higher thresholds than thermodynamic calculations.

In this general framework, a reasoned review of the critical energy equations readily available in the literature, and the related expressions of the critical bubble radius, is carried out. A pair of relationships for the determination of the critical energy and bubble radius are then proposed and discussed.

\section{Critical energy for bubble nucleation}

A liquid at temperature $T_{L}$ and pressure $\mathrm{p}_{\mathrm{L}}$ is called superheated when $T_{L}$ is higher than the saturation temperature $T_{V}$ at pressure $\mathrm{p}_{\mathrm{L}}$, or, that is the same, $\mathrm{p}_{\mathrm{L}}$ is lower than the saturation pressure $\mathrm{p}_{\mathrm{V}}$ at temperature $\mathrm{T}_{\mathrm{L}}$, as shown in the $\mathrm{pT}$ phase diagram depicted in Fig. 1, in which the saturation line separating the vapour and liquid single-phase regions represents the two-phase liquid-vapour region. It can be seen that for 


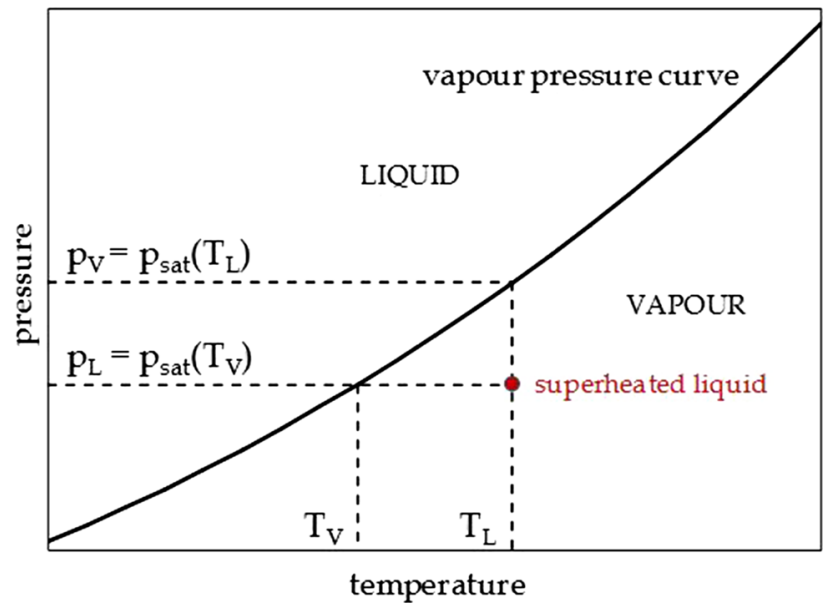

Fig. 1 pT phase diagram for the liquid and vapour regions

each saturation pressure there is a unique saturation temperature and vice versa, their correspondence being described by the Clapeyron-Clausius equation

$\frac{\mathrm{dp}}{\mathrm{dT}}=\frac{\rho_{\mathrm{V}} \lambda}{\mathrm{T}\left(1-\rho_{\mathrm{V}} / \rho_{\mathrm{L}}\right)}$,

where $\lambda$ is the latent heat of vaporization, and $\rho_{\mathrm{L}}$ and $\rho_{\mathrm{V}}$ are the mass densities of the saturated liquid and vapour phases.

Notice that, strictly speaking, the metastable liquid state of coordinates $\left(\mathrm{T}_{\mathrm{L}}, \mathrm{p}_{\mathrm{L}}\right)$, which apparently falls in the vapour region, could not be displayed in the pT phase diagram, wherein only stable equilibrium states can be represented. Of course, the degree of metastability of the superheated liquid can be expressed either in terms of superheat, $\Delta \mathrm{T}=\mathrm{T}_{\mathrm{L}}-\mathrm{T}_{\mathrm{V}}$, or in terms of underpressure, $\Delta \mathrm{p}=\mathrm{p}_{\mathrm{V}}-\mathrm{p}_{\mathrm{L}}$.

In a bubble chamber in which the sensitive liquid is kept superheated at temperature $\mathrm{T}_{\mathrm{L}}$ and pressure $\mathrm{p}_{\mathrm{L}}$, if enough energy is deposited into the liquid, the formation of a critically-sized vapour bubble occurs, its radius $R_{c}$ being given by the Young-Laplace relation

$\mathrm{R}_{\mathrm{c}}=\frac{2 \sigma}{\mathrm{p}_{\mathrm{b}}-\mathrm{p}_{\mathrm{L}}}$,

where $\sigma$ is the surface tension of the liquid, and $\mathrm{p}_{\mathrm{b}}$ is the pressure inside the bubble.

The critical energy $E_{c}$ required for bubble nucleation has been the subject of a number of studies conducted in the past, each leading to an expression composed of different terms. The terms commonly included in the critical energy equation are the energy required to vaporize the mass of liquid involved in the phase change and the energy required to form the bubble surface. In many formulations an expansion term is added, mostly to account for the expansion work transferred from the vapour bubble to the surrounding liquid during the vapour bubble formation, while less frequently a term accounting for the kinetic energy imparted by the expanding vapour bubble to the surrounding liquid is also incorporated. A list of the originally proposed equations are summarized in Table 1, in which $\mathrm{v}_{\mathrm{r}}$ denotes the radial velocity of expansion of the bubble surface, whereas all the other variables have already been defined earlier throughout the text. Notice that all the physical properties are typically calculated at the liquid temperature $\mathrm{T}_{\mathrm{L}}$.

Actually, the critical energy is completely described by two terms: the vaporization term, and the surface formation term expressed in the form first introduced by Bugg [10]. In fact, based on the first law of thermodynamics, the heat injection required to nucleate a critical bubble, i.e., the critical energy $E_{c}$, is given by the sum of the internal energy variation, $\Delta \mathrm{U}$, and the expansion work $\mathrm{W}_{\text {exp }}$ transferred from the vapour bubble to the surrounding liquid during the vapour bubble formation

$\mathrm{E}_{\mathrm{c}}=\Delta \mathrm{U}+\mathrm{W}_{\exp }$.

On the other hand, once the vapour bubble is thought as composed of its bulk volume and the interfacial region, conventionally assumed to have no thickness and thus represented by the mathematical surface of the bubble, $\Delta \mathrm{U}$ can be written as the sum of a volume term, $\Delta \mathrm{U}_{\mathrm{vol}}$, and a surface term, $\Delta \mathrm{U}_{\text {surf }}$, giving

$\mathrm{E}_{\mathrm{c}}=\Delta \mathrm{U}_{\mathrm{vol}}+\Delta \mathrm{U}_{\text {surf }}+\mathrm{W}_{\text {exp }}$.

This means that, if the internal energy variation $\Delta U_{\text {vol }}$ from the metastable liquid state to the stable saturated vapour state is approximated using the difference between the internal energies of the stable saturated vapour and liquid states at pressure $\mathrm{p}_{\mathrm{L}}$, then, according to the definition of the latent heat of vaporization based on the first law of thermodynamics, the sum $\Delta \mathrm{U}_{\mathrm{vol}}+\mathrm{W}_{\text {exp }}$ equals the heat $\mathrm{Q}_{\mathrm{evap}}$ required for the phase change to occur at the constant pressure $\mathrm{p}_{\mathrm{L}}$ [31], i.e.,

$\mathrm{E}_{\mathrm{c}}=\mathrm{Q}_{\text {evap }}+\Delta \mathrm{U}_{\text {surf }}$.

The same conclusion can be achieved by simply considering that, based on the definition of enthalpy as $\mathrm{H}=\mathrm{U}+\mathrm{pV}$, the injection of $E_{c}$ at the constant pressure $p_{L}$ results in an enthalpy variation $\Delta \mathrm{H}$, which can be written as the sum of a volume term, $\Delta \mathrm{H}_{\mathrm{vol}}$, and a surface term, $\Delta \mathrm{H}_{\text {surf }}$. The $\Delta \mathrm{H}_{\mathrm{vol}}$ term can be approximated by the heat $\mathrm{Q}_{\text {evap }}$ required for the phase change at the liquid pressure $\mathrm{p}_{\mathrm{L}}$, whereas the $\Delta \mathrm{H}_{\text {surf }}$ term, on account of the definition of enthalpy cited earlier, coincides with the internal energy variation $\Delta \mathrm{U}_{\text {surf }}$, since the bubble surface region has no volume.

The heat of vaporization is given by

$\mathrm{Q}_{\text {evap }}=\frac{4}{3} \pi \mathrm{R}_{\mathrm{c}}^{3} \rho_{\mathrm{V}} \lambda$,

in which, therefore, both $\rho_{\mathrm{V}}$ and $\lambda$ should be calculated at the stable equilibrium temperature at which the phase change 
Table 1 Terms in the $E_{c}$ equation proposed by different authors

\begin{tabular}{|c|c|c|c|c|c|c|}
\hline Author(s) & Year & Vaporization & Surface formation & Expansion & Kinetic energy & See also Refs. \\
\hline Pless and Plano [9] & 1956 & $\frac{4}{3} \pi \mathrm{R}_{\mathrm{c}}^{3} \rho_{\mathrm{V}} \lambda$ & $4 \pi \mathrm{R}_{\mathrm{c}}^{2} \sigma$ & $\frac{4}{3} \pi \mathrm{R}_{\mathrm{c}}^{3} \mathrm{p}_{\mathrm{L}}$ & - & \\
\hline Seitz [8] & 1958 & $\frac{4}{3} \pi \mathrm{R}_{\mathrm{c}}^{3} \rho_{\mathrm{V}} \lambda$ & $4 \pi \mathrm{R}_{\mathrm{c}}^{2} \sigma$ & - & - & {$[15,16]$} \\
\hline Bugg [10] & 1959 & $\frac{4}{3} \pi \mathrm{R}_{\mathrm{c}}^{3} \rho_{\mathrm{V}} \lambda$ & $4 \pi \mathrm{R}_{\mathrm{c}}^{2}\left(\sigma-\frac{\mathrm{d} \sigma}{\mathrm{dT}} \mathrm{T}_{\mathrm{L}}\right)$ & $-\frac{4}{3} \pi \mathrm{R}_{\mathrm{c}}^{3}\left(\mathrm{p}_{\mathrm{V}}-\mathrm{p}_{\mathrm{L}}\right)$ & - & {$[3,17-22]$} \\
\hline Norman and Spiegler [11] & 1963 & $\frac{4}{3} \pi \mathrm{R}_{\mathrm{c}}^{3} \rho_{\mathrm{V}} \lambda$ & $4 \pi \mathrm{R}_{\mathrm{c}}^{2}\left(\sigma-\frac{\mathrm{d} \sigma}{\mathrm{d} \mathrm{T}} \mathrm{T}_{\mathrm{L}}\right)$ & - & $2 \pi \rho_{\mathrm{L}} \mathrm{R}_{\mathrm{c}}{ }^{3} \mathrm{v}_{\mathrm{r}}^{2}$ & \\
\hline Tenner [12] & 1963 & $\frac{4}{3} \pi \mathrm{R}_{\mathrm{c}}^{3} \rho_{\mathrm{V}} \lambda$ & $4 \pi \mathrm{R}_{\mathrm{c}}^{2}\left(\sigma-\frac{\mathrm{d} \sigma}{\mathrm{dT}} \mathrm{T}_{\mathrm{L}}\right)$ & $\frac{4}{3} \pi \mathrm{R}_{\mathrm{c}}^{3}\left(1-\frac{\rho_{\mathrm{V}}}{\rho_{\mathrm{L}}}\right) \mathrm{p}_{\mathrm{L}}$ & - & \\
\hline Peyrou [13] & 1967 & $\frac{4}{3} \pi \mathrm{R}_{\mathrm{c}}^{3} \rho_{\mathrm{V}} \lambda$ & $4 \pi \mathrm{R}_{\mathrm{c}}^{2}\left(\sigma-\frac{\mathrm{d} \sigma}{\mathrm{dT}} \mathrm{T}_{\mathrm{L}}\right)$ & $\frac{4}{3} \pi \mathrm{R}_{\mathrm{c}}^{3} \mathrm{p}_{\mathrm{L}}$ & - & {$[23-27]$} \\
\hline Bell et al. [14] & 1974 & $\frac{4}{3} \pi \mathrm{R}_{\mathrm{c}}^{3} \rho_{\mathrm{V}} \lambda$ & $4 \pi \mathrm{R}_{\mathrm{c}}^{2} \sigma$ & $-\frac{4}{3} \pi \mathrm{R}_{\mathrm{c}}^{3}\left(\mathrm{p}_{\mathrm{V}}-\mathrm{p}_{\mathrm{L}}\right)$ & $2 \pi \rho_{\mathrm{L}} \mathrm{R}_{\mathrm{c}}^{3} \mathrm{v}_{\mathrm{r}}^{2}$ & [28-30] \\
\hline
\end{tabular}

takes place at the constant pressure $\mathrm{p}_{\mathrm{L}}$, that is to say, the saturation temperature $\mathrm{T}_{\mathrm{V}}$. Further considerations on the temperature at which both $\rho_{\mathrm{V}}$ and $\lambda$ should be evaluated will be mentioned later.

The internal energy variation of the bubble surface $\Delta U_{\text {surf }}$ can be calculated considering that the energy required to form the bubble surface is expressed in terms of the free energy, whose variation associated with the formation of a unit surface area equals the surface tension of the liquid, $\sigma=\mathrm{dF} / \mathrm{dA}$. Therefore, on account of the definition of free energy as $\mathrm{F}=\mathrm{U}-\mathrm{TS}$, the internal energy variation consequent to the formation of the bubble surface at the constant temperature $\mathrm{T}_{\mathrm{L}}$ can be written as

$$
\Delta \mathrm{U}_{\text {surf }}=\Delta \mathrm{F}_{\text {surf }}+\mathrm{T}_{\mathrm{L}} \Delta \mathrm{S}_{\text {surf }}
$$

in which, since the bubble surface grows from 0 to $4 \pi \mathrm{R}_{\mathrm{c}}^{2}$, the variation of any state function clearly coincides with the value of the state function at the end of the bubble formation. According to the first law of thermodynamics, if (7) is rewritten as $\Delta \mathrm{U}_{\text {surf }}=\mathrm{T}_{\mathrm{L}} \Delta \mathrm{S}_{\text {surf }}-\left(-\Delta \mathrm{F}_{\text {surf }}\right)$, then $\mathrm{T}_{\mathrm{L}} \Delta \mathrm{S}_{\text {surf }}$ represents the heat that must be supplied to the bubble surface to keep it at the constant temperature $T_{L}$, whereas $-\Delta F_{\text {surf }}$ is the isothermal work done by the bubble surface during its formation, or better, $\Delta \mathrm{F}_{\text {surf }}$ is the work that must be supplied to the bubble surface to allow its formation.

The free energy variation $\Delta \mathrm{F}_{\text {surf }}$ is given by the product of the area of the bubble surface multiplied by the surface tension

$\Delta \mathrm{F}_{\text {surf }}=4 \pi \mathrm{R}_{\mathrm{c}}^{2} \sigma$

The entropy variation $\Delta S_{\text {surf }}$, computed in terms of the entropy of the bubble surface at the end of its formation $S_{\text {surf }}$, is given by minus the temperature derivative of the surface free energy $F_{\text {surf }}$ calculated using (8). In fact, on account of the first and second laws of thermodynamics, the differential $\mathrm{dF}$ is

$\mathrm{dF}=-\mathrm{pdV}-\mathrm{SdT}$, suggesting

$\mathrm{F}=\mathrm{F}(\mathrm{V}, \mathrm{T})$,

$\mathrm{dF}=\left(\frac{\partial \mathrm{F}}{\partial \mathrm{V}}\right)_{\mathrm{T}} \mathrm{dV}+\left(\frac{\partial \mathrm{F}}{\partial \mathrm{T}}\right)_{\mathrm{V}} \mathrm{dT}$,

$-\left(\frac{\partial \mathrm{F}}{\partial \mathrm{V}}\right)_{\mathrm{T}}=\mathrm{p}$,

$-\left(\frac{\partial \mathrm{F}}{\partial \mathrm{T}}\right)_{\mathrm{V}}=\mathrm{S}$.

Notice that, since the bubble surface region has no volume, the free energy of the bubble surface is a function of the temperature only, which results in $\mathrm{S}_{\text {surf }}=-\mathrm{dF}_{\text {surf }} / \mathrm{dT}$, thus implying

$$
\Delta \mathrm{S}_{\text {surf }}=-4 \pi \mathrm{R}_{\mathrm{c}}{ }^{2} \frac{\mathrm{d} \sigma}{\mathrm{dT}} .
$$

The combination of (7), (8) and (14) gives

$\Delta \mathrm{U}_{\text {surf }}=4 \pi \mathrm{R}_{\mathrm{c}}{ }^{2}\left(\sigma-\mathrm{T}_{\mathrm{L}} \frac{\mathrm{d} \sigma}{\mathrm{dT}}\right)$.

Of course, the same relation (15) can also be achieved using other ways, see, e.g., [32].

Substituting (6) and (15) in (5), we obtain

$\mathrm{E}_{\mathrm{c}}=\frac{4}{3} \pi \mathrm{R}_{\mathrm{c}}^{3} \rho_{\mathrm{V}} \lambda+4 \pi \mathrm{R}_{\mathrm{c}}^{2}\left(\sigma-\mathrm{T}_{\mathrm{L}} \frac{\mathrm{d} \sigma}{\mathrm{dT}}\right)$.

Accordingly, neither the expansion term nor the kinetic energy term have to be included in the expression of the critical energy. In particular, as seen earlier, the expansion work done by the vapour bubble during its formation is already comprised in the vaporization term. As a matter of fact, the vaporization term consists of both the energy required to break the intermolecular bonds in the liquid, which results in an increased internal energy of the vapour phase, and the energy required to draw the vapour molecules apart, which corresponds to the positive expansion work transferred to the liquid. Thus, all the authors who add an expansion work in the critical energy equation assume that the heat required for the evaporation of the liquid is responsible only for the volume internal energy increase. On the other hand, some 
authors subtract an expansion term which, assuming that the radius of the vapour bubble grows from 0 to $R_{c}$, represents the difference between the expansion work executed at the vapour pressure $\mathrm{p}_{\mathrm{V}}$ and the expansion work executed at the liquid pressure $\mathrm{p}_{\mathrm{L}}$, thus resulting in a correction of the vaporization term. In fact, owing to this subtraction, the expansion work per unit mass included in the latent heat of vaporization becomes referred to the equilibrium vapour temperature $T_{V}$ rather than to the liquid temperature $\mathrm{T}_{\mathrm{L}}$. Finally, the kinetic energy of the liquid, being the same as the net work done on the liquid once any irreversibility effect is neglected, needs not to be considered.

All in all, the equation proposed by Bugg [10] (third line of Table 1), which includes the subtractive expansion term, disregarding at the same time the kinetic energy term, seems to be essentially equivalent to the proposed equation (16), at least as long as the values of the specific internal energy variation and the mass density of the saturated vapour calculated at temperature $T_{L}$ are not too different from those calculated at temperature $T_{V}$, i.e., the superheat degree is not too high.

\section{Radius of the critically-sized nucleated vapour bubble}

The radius $R_{c}$ of the critically-sized vapour bubble given by (2) is normally calculated in the hypothesis of stable equilibrium conditions, despite this is not the real situation. Indeed, pressure $\mathrm{p}_{\mathrm{b}}$ is usually approximated using the saturation pressure at the liquid temperature [8-11,13-16,18,20,22-30], which gives

$\mathrm{R}_{\mathrm{c}} \approx \frac{2 \sigma}{\mathrm{p}_{\mathrm{V}}-\mathrm{p}_{\mathrm{L}}}$.

Differently, some authors $[3,12,17,19,21]$ approximate $\mathrm{p}_{\mathrm{b}}$ using the pressure value obtained by imposing the stable equilibrium condition of equality of the chemical potentials, or, that is the same, the specific free enthalpies of the metastable liquid and the stable vapour at the liquid temperature, and assuming that the mass densities of the liquid and vapour phases are substantially the same as their corresponding saturation values at the liquid temperature, which results in

$\mathrm{R}_{\mathrm{c}} \approx \frac{2 \sigma}{\left(\mathrm{p}_{\mathrm{V}}-\mathrm{p}_{\mathrm{L}}\right)\left(1-\frac{\rho_{\mathrm{V}}}{\rho_{\mathrm{L}}}\right)}$.

Actually, although both mentioned approximations can be considered as reasonably true at low degrees of metastability, their application at the high superheats asked for WIMPrecoil detection can lose accuracy. In this regard, an alternative approach can be followed by recalling that, when a thermodynamic system kept at constant temperature and pressure can be in more than one equilibrium state, then the stable equilibrium state is the state of lowest free enthalpy,

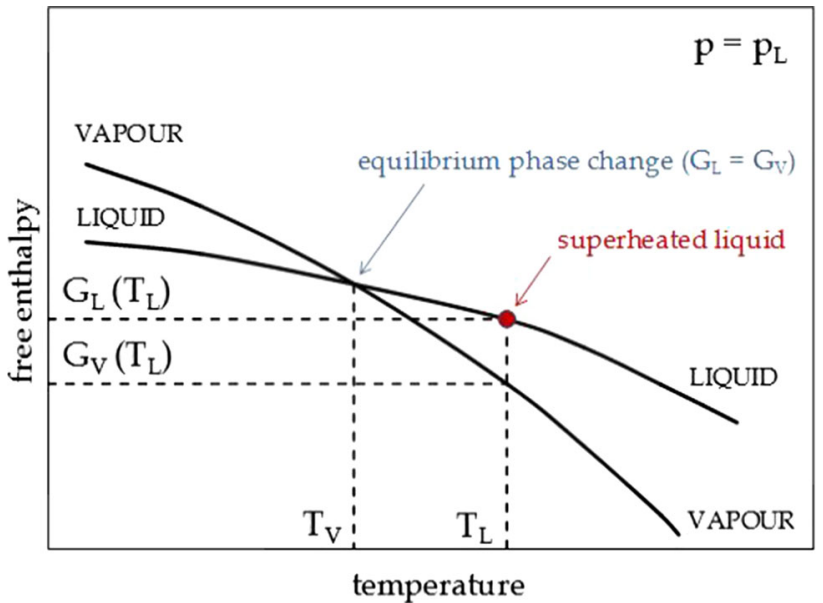

Fig. 2 Distributions of free enthalpies $\mathrm{G}_{\mathrm{L}}$ and $\mathrm{G}_{\mathrm{V}}$ vs. $\mathrm{T}$ at $\mathrm{p}=\mathrm{p}_{\mathrm{L}}$

also named Gibbs free energy and defined as $\mathrm{G}=\mathrm{H}-\mathrm{TS}$, which therefore plays the same role played by the potential energy in defining the stable equilibrium state of a mechanical system [31]. It follows that in the present case the free enthalpy of the superheated liquid $\mathrm{G}_{\mathrm{L}}$ is necessarily higher than that of the stable vapour $\mathrm{GV}_{\mathrm{V}}$ of an amount $\Delta \mathrm{G}$ that can also be seen as the free enthalpy variation associated with the formation of a vapour bubble. The situation is schematically displayed in Fig. 2, where typical distributions of $\mathrm{G}_{\mathrm{L}}$ and $G_{V}$ at the constant pressure $\mathrm{p}_{\mathrm{L}}$ are plotted versus $\mathrm{T}$. It is apparent that, since $\Delta \mathrm{G}$ increases as the metastability degree is increased, the assumption of equality of $\mathrm{G}_{\mathrm{L}}$ and $\mathrm{G}_{\mathrm{V}}$ may become inaccurate at high degrees of superheat.

Thus, a more realistic approach is required which should be able to reflect that the critical size represents a condition of absolute instability for the vapour bubble. In fact, should the critically-sized vapour bubble lose just a tiny amount of matter, say one molecule, which gets back to be part of the surrounding liquid, then the bubble will literally implode, vanishing, due to the loss of the mechanical equilibrium. Conversely, should the critically-sized vapour bubble gain just a tiny amount of matter, taken away from the surrounding liquid, then the bubble will spontaneously grow, becoming detectable.

In view of the mentioned relation between the value of the free enthalpy and the stability of a system that can be in more than one equilibrium state at constant $\mathrm{T}$ and $\mathrm{p}$, such an extreme instability condition must correspond to a maximum of the difference between the free enthalpies of the superheated liquid and the stable vapour, or, that is the same, a maximum of the free enthalpy variation associated with the phase change, which is an approach also used in the study of crystal nucleation, see, e.g., [33-36]. The critical radius $\mathrm{R}_{\mathrm{c}}$ can then be regarded as the size of the vapour bubble corresponding to the maximum of the function which describes the 
free enthalpy variation $\Delta G(r)$ associated with the formation of a vapour bubble of radius $r$ that nucleates in a metastable liquid kept at constant temperature $\mathrm{T}_{\mathrm{L}}$ and pressure $\mathrm{p}_{\mathrm{L}}$

$$
\Delta \mathrm{G}(\mathrm{r})=\Delta \mathrm{H}(\mathrm{r})-\mathrm{T}_{\mathrm{L}} \Delta \mathrm{S}(\mathrm{r}),
$$

which can also be written as the sum of a volume term $\Delta \mathrm{G}_{\mathrm{vol}}(\mathrm{r})$ and a surface term $\Delta \mathrm{G}_{\text {surf }}(\mathrm{r})$ giving

$$
\Delta \mathrm{G}(\mathrm{r})=\Delta \mathrm{G}_{\mathrm{vol}}(\mathrm{r})+\Delta \mathrm{G}_{\text {surf }}(\mathrm{r}) .
$$

The volume term can be expressed as

$$
\Delta \mathrm{G}_{\mathrm{vol}}(\mathrm{r})=\Delta \mathrm{H}_{\mathrm{vol}}(\mathrm{r})-\mathrm{T}_{\mathrm{L}} \Delta \mathrm{S}_{\mathrm{vol}}(\mathrm{r}),
$$

where, as previously done for the internal energy variation, the enthalpy and entropy variations from the metastable liquid state to the stable vapour state can be approximated using the respective stable equilibrium variations at temperature $\mathrm{T}_{\mathrm{V}}$, provided that the superheat degree is sufficiently small compared with the difference between the critical and triple points. Actually, this is an easy way to estimate $\Delta \mathrm{H}_{\mathrm{vol}}(\mathrm{r})$ and $\Delta S_{\mathrm{vol}}(r)$, whose values would be otherwise difficult to determine, and to account for the metastability degree in the derivation of $\Delta \mathrm{G}(\mathrm{r})$. Therefore, $\Delta \mathrm{H}_{\mathrm{vol}}(\mathrm{r})$ and $\Delta \mathrm{S}_{\mathrm{vol}}(\mathrm{r})$ are calculated as the heat required for the phase change at temperature $T_{V}$, and the heat required for the phase change at temperature $\mathrm{T}_{\mathrm{V}}$ divided by the same temperature $\mathrm{T}_{\mathrm{V}}$, respectively

$$
\begin{aligned}
\Delta \mathrm{H}_{\mathrm{vol}}(\mathrm{r}) & =\frac{4}{3} \pi \mathrm{r}^{3} \rho_{\mathrm{V}} \lambda, \\
\Delta \mathrm{S}_{\mathrm{vol}}(\mathrm{r}) & =\frac{\frac{4}{3} \pi \mathrm{r}^{3} \rho_{\mathrm{V}} \lambda}{\mathrm{T}_{\mathrm{V}}},
\end{aligned}
$$

in which both $\rho_{\mathrm{V}}$ and $\lambda$ must be evaluated at temperature $\mathrm{T}_{\mathrm{V}}$. Hence

$$
\Delta \mathrm{G}_{\mathrm{vol}}(\mathrm{r})=\frac{4}{3} \pi \mathrm{r}^{3} \rho_{\mathrm{V}} \lambda\left(1-\frac{\mathrm{T}_{\mathrm{L}}}{\mathrm{T}_{\mathrm{V}}}\right) .
$$

As far as the surface term is concerned, based on the cited definitions of enthalpy and free energy, the free enthalpy can be expressed as $\mathrm{G}=\mathrm{F}+\mathrm{pV}$, thus following that, since the bubble surface region has no volume, the free enthalpy change $\Delta \mathrm{G}_{\text {surf }}(\mathrm{r})$ coincides with the free energy change $\Delta \mathrm{F}_{\text {surf }}(\mathrm{r})$, which can be directly derived from (8) by simply replacing $R_{c}$ with $r$, i.e.,

$\Delta \mathrm{G}_{\text {surf }}(\mathrm{r})=4 \pi \mathrm{r}^{2} \sigma$.

The combination of (20), (24) and (25) gives

$$
\Delta \mathrm{G}(\mathrm{r})=-\frac{4}{3} \pi \mathrm{r}^{3} \rho_{\mathrm{V}} \lambda \frac{\mathrm{T}_{\mathrm{L}}-\mathrm{T}_{\mathrm{V}}}{\mathrm{T}_{\mathrm{V}}}+4 \pi \mathrm{r}^{2} \sigma .
$$

Indeed, (26) can also be obtained by determining $\Delta \mathrm{H}(\mathrm{r})$ and $\Delta \mathrm{S}(\mathrm{r})$, and substituting their expressions in (19).

The enthalpy variation $\Delta \mathrm{H}(\mathrm{r})$, equal to the heat injection required to nucleate the bubble, can be directly derived from
(16), by simply replacing $R_{c}$ with $r$, i.e.,

$$
\Delta \mathrm{H}(\mathrm{r})=\frac{4}{3} \pi \mathrm{r}^{3} \rho_{\mathrm{V}} \lambda+4 \pi \mathrm{r}^{2}\left(\sigma-\mathrm{T}_{\mathrm{L}} \frac{\mathrm{d} \sigma}{\mathrm{dT}}\right) .
$$

On the other hand, the entropy variation $\Delta S(r)$ can be written as the sum of a volume term, $\Delta \mathrm{S}_{\mathrm{vol}}(\mathrm{r})$, and a surface term, $\Delta \mathrm{S}_{\text {surf }}(\mathrm{r})$. The volume term $\Delta \mathrm{S}_{\mathrm{vol}}(\mathrm{r})$ is given by (23), whereas the surface term $\Delta S_{\text {surf }}(r)$ can be directly derived from (14), by simply replacing $R_{c}$ with $r$, thus obtaining

$$
\Delta \mathrm{S}(\mathrm{r})=\frac{\frac{4}{3} \pi \mathrm{r}^{3} \rho_{\mathrm{V}} \lambda}{\mathrm{T}_{\mathrm{V}}}-4 \pi \mathrm{r}^{2} \frac{\mathrm{d} \sigma}{\mathrm{dT}}
$$

It seems worth pointing out that, since (26) has been obtained by evaluating both $\rho_{\mathrm{V}}$ and $\lambda$ at temperature $\mathrm{T}_{\mathrm{V}}$, also in (27) both $\rho_{\mathrm{V}}$ and $\lambda$ must be evaluated at temperature $\mathrm{T}_{\mathrm{V}}$. On the other hand, since (27) has been directly derived from (16), then also in (16) both $\rho_{\mathrm{V}}$ and $\lambda$ should be evaluated at temperature $\mathrm{T}_{\mathrm{V}}$, as already stated in the previous paragraph. Conversely, both $\sigma$ and $\mathrm{d} \sigma / \mathrm{dT}$ have to be calculated at temperature $T_{L}$.

The critical radius of the vapour bubble, $R_{c}$, is then determined by computing the root of the derivative $\Delta \mathrm{G}^{\prime}(\mathrm{r})$, again assuming that $\sigma$ is independent of the vapour bubble curvature [17], which results in

$\mathrm{R}_{\mathrm{c}}=\frac{2 \sigma}{\rho_{\mathrm{V}} \lambda \frac{\mathrm{T}_{\mathrm{L}}-\mathrm{T}_{\mathrm{V}}}{\mathrm{T}_{\mathrm{V}}}}$.

Hence, the formation of a vapour bubble occurs via a pathway involving the surmounting of the barrier of potential $\Delta G\left(R_{c}\right)$, whose value is given by (26) with $r=R_{c}$. A number of distributions of $\Delta G(r)$ relative to $\mathrm{C}_{3} \mathrm{~F}_{8}$, i.e., the target liquid used for WIMP-recoil detection in the experiments carried out by PICO [6] and MOSCAB [7], are plotted in Fig. 3 against the radius $r$ for $T_{L}=20^{\circ} \mathrm{C}$ using the superheat degree $\Delta \mathrm{T}$ as a parameter. The values of the physical properties are taken from the NIST Chemistry WebBook [37].

Of course, should the degree of metastability of the superheated liquid be sufficiently low, then (1) can be rewritten by approximating the temperature derivative $\mathrm{dp} / \mathrm{dT}$ with the corresponding increment ratio, i.e., the ratio between the underpressure $\Delta \mathrm{p}$ and the superheat $\Delta \mathrm{T}$, thus obtaining

$\frac{\mathrm{p}_{\mathrm{V}}-\mathrm{p}_{\mathrm{L}}}{\mathrm{T}_{\mathrm{L}}-\mathrm{T}_{\mathrm{V}}} \approx \frac{\rho_{\mathrm{V}} \lambda}{\mathrm{T}_{\mathrm{V}}\left(1-\rho_{\mathrm{V}} / \rho_{\mathrm{L}}\right)}$

and then

$\rho_{\mathrm{V}} \lambda \frac{\mathrm{T}_{\mathrm{L}}-\mathrm{T}_{\mathrm{V}}}{\mathrm{T}_{\mathrm{V}}} \approx\left(\mathrm{p}_{\mathrm{V}}-\mathrm{p}_{\mathrm{L}}\right)\left(1-\frac{\rho_{\mathrm{V}}}{\rho_{\mathrm{L}}}\right)$,

in which, due to the low $\Delta \mathrm{T}$, the density ratio at temperatures $\mathrm{T}_{\mathrm{V}}$ and $\mathrm{T}_{\mathrm{L}}$ is practically the same. The replacement of (31) in (29) leads to (18). Moreover, if we take into account that 


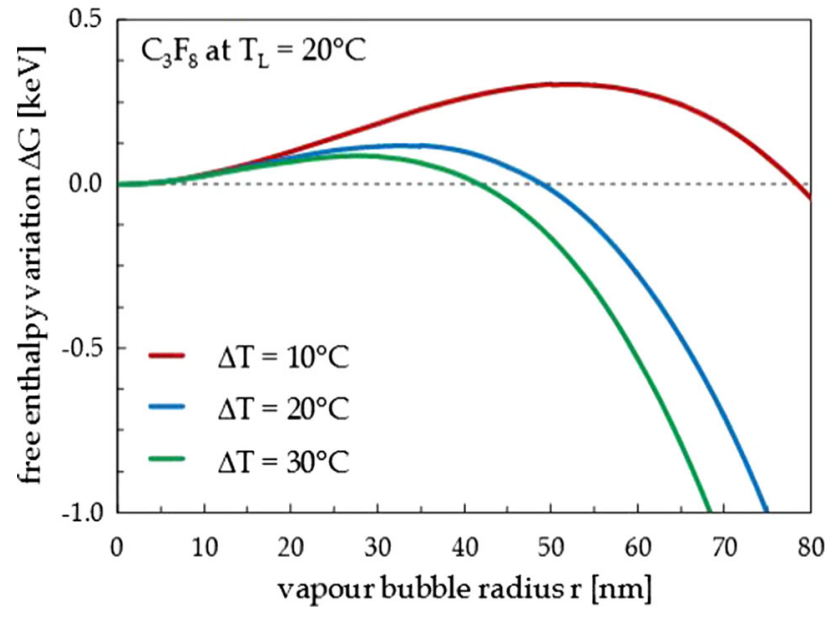

Fig. 3 Distributions of $\Delta \mathrm{G}(\mathrm{r})$ vs. $\mathrm{r}$ for $\mathrm{C}_{3} \mathrm{~F}_{8}$ at $\mathrm{T}_{\mathrm{L}}=20^{\circ} \mathrm{C}$, using $\Delta \mathrm{T}$ as a parameter

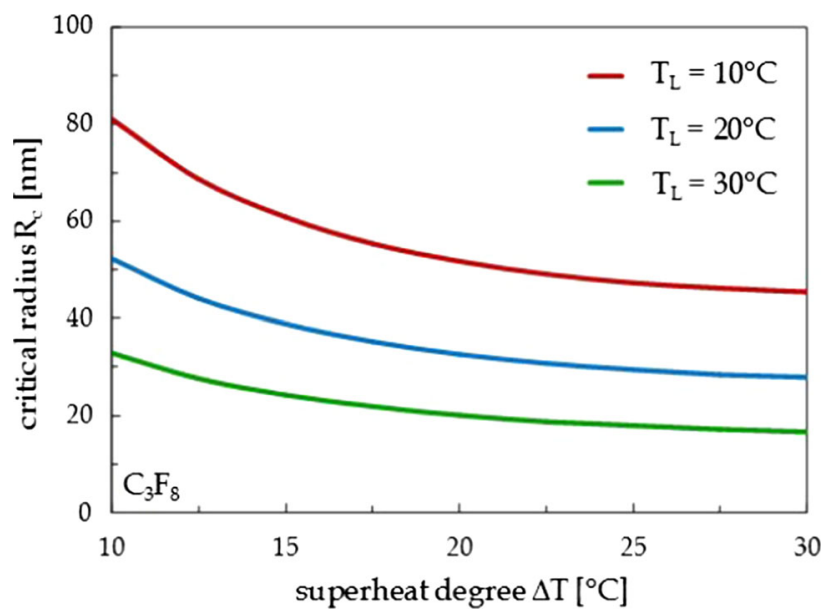

Fig. 4 Distributions of $R_{c}$ vs. $\Delta T$ for $C_{3} F_{8}$ using $T_{L}$ as a parameter

$\rho_{\mathrm{V}} / \rho_{\mathrm{L}} \ll 1$, (31) can be further reduced to

$\rho_{\mathrm{V}} \lambda \frac{\mathrm{T}_{\mathrm{L}}-\mathrm{T}_{\mathrm{V}}}{\mathrm{T}_{\mathrm{V}}} \approx \mathrm{p}_{\mathrm{V}}-\mathrm{p}_{\mathrm{L}}$,

which, replaced in (29), leads to (17).

Two sets of distributions of the critical radius expressed by (29) and the related critical energy expressed by (16), plotted against the superheat degree using the liquid temperature as a parameter, are reported in Figs. 4 and 5 for $\mathrm{C}_{3} \mathrm{~F}_{8}$. Notice that the uncertainty on the values of the critical energy can be estimated to be of the order of 6-7\%, due to the uncertainties on the physical properties, primarily the surface tension.

\section{Discussion}

First of all, it is worth observing that the procedure followed to obtain (29) by determining $\Delta \mathrm{H}(\mathrm{r})$ and $\Delta \mathrm{S}(\mathrm{r})$, and then substituting their expressions in (19), intrinsically demon-

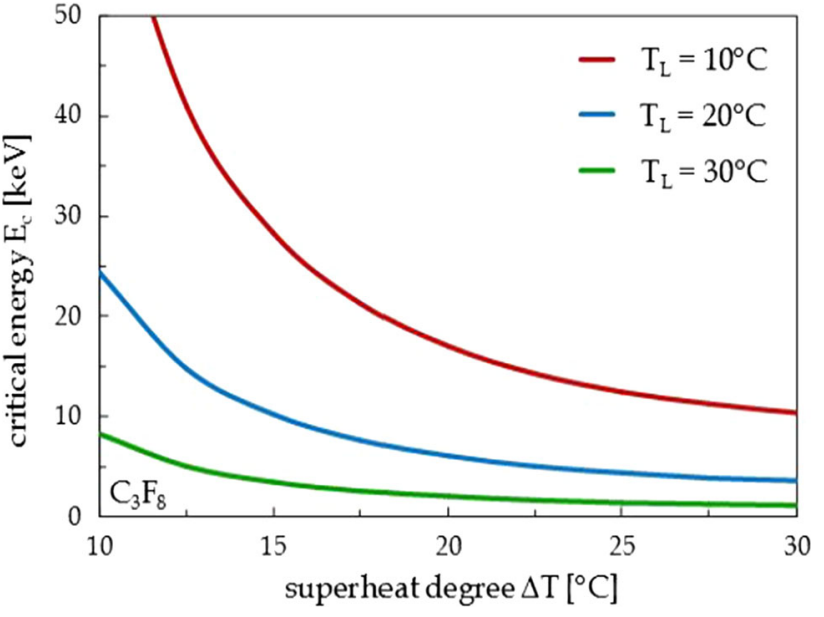

Fig. 5 Distributions of $\mathrm{E}_{\mathrm{c}}$ vs. $\Delta \mathrm{T}$ for $\mathrm{C}_{3} \mathrm{~F}_{8}$ using $\mathrm{T}_{\mathrm{L}}$ as a parameter

strates the validity of (16). In fact, should the heat injection required to nucleate a vapour bubble have been derived from a relationship different from (16), then a relationship different from (29) would have been achieved for the critical radius $\mathrm{R}_{\mathrm{c}}$, and neither (18) nor (17) could have been obtained for low degrees of metastability.

Furthermore, it must be pointed out that the calculation of the critical radius $\mathrm{R}_{\mathrm{c}}$ by the way of (17) or (18) leads to values lower than that expressed by (29), which is a direct consequence of the fact that, since the vapour pressure curve is concave upwards, the temperature derivative of the saturation pressure at temperature $T_{V}$ is lower than the corresponding increment ratio $\left(\mathrm{p}_{\mathrm{V}}-\mathrm{p}_{\mathrm{L}}\right) /\left(\mathrm{T}_{\mathrm{L}}-\mathrm{T}_{\mathrm{V}}\right)$. Of course, the discrepancy increases as the degree of metastability is increased, as shown in Fig. 6, in which a number of distributions of the relative difference $\delta_{R}=\left(R_{c}-R_{c}^{*}\right) / R_{c}$ between the results obtained applying (18) instead of (29) are plotted against the superheat degree $\Delta \mathrm{T}$ for $\mathrm{C}_{3} \mathrm{~F}_{8}$ using the liquid temperature $T_{L}$ as a parameter, where $R_{c}$ and $R_{c}^{*}$ are the values of the critical radius given by (29) and (18), respectively. Even higher discrepancies are obtained if (17) is applied rather than (18).

Accordingly, the critical energy obtained through (16) in which $R_{c}$ is calculated by (29) is higher than the critical energy derived applying, for example, the equation proposed by Bugg [10] using (18) to calculate $\mathrm{R}_{\mathrm{c}}$.

A set of distributions of the relative difference $\delta_{\mathrm{E}}=$ $\left(\mathrm{E}_{\mathrm{c}}-\mathrm{E}_{\mathrm{c}}^{*}\right) / \mathrm{E}_{\mathrm{c}}$ between the results obtained applying the Bugg's equation in combination with (18), instead of (16) in combination with (29), are plotted in Fig. 7 against the superheat degree $\Delta \mathrm{T}$ for $\mathrm{C}_{3} \mathrm{~F}_{8}$ using the liquid temperature $\mathrm{T}_{\mathrm{L}}$ as a parameter, where $\mathrm{E}_{\mathrm{c}}$ and $\mathrm{E}_{\mathrm{c}}^{*}$ are the values of the critical energy given by (16) and by the Bugg's equation, respectively. It is apparent that when the degree of metastability of the superheated liquid is high enough, the relative difference 


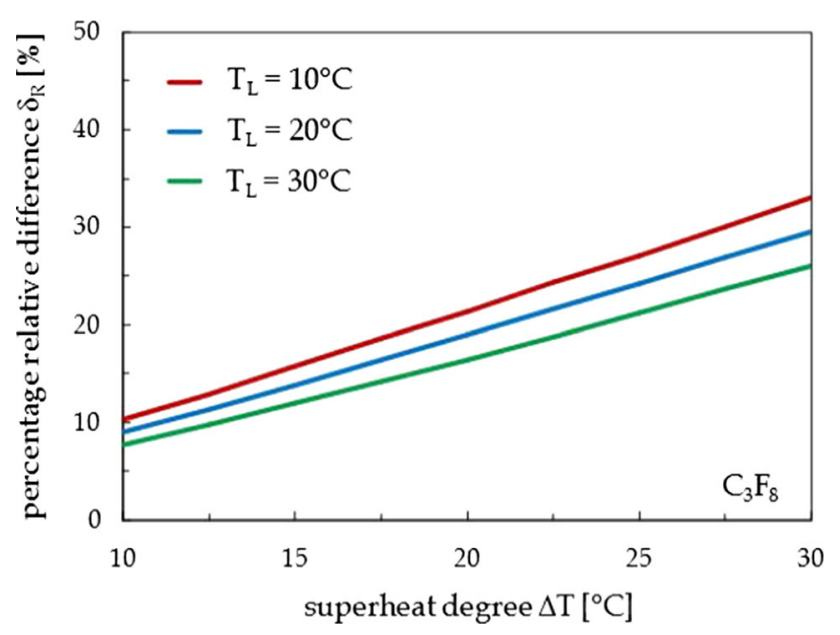

Fig. 6 Distributions of $\delta_{R}=\left(R_{c}-R_{c}^{*}\right) / R_{c}$ vs. $\Delta T$ for $C_{3} F_{8}$ using $T_{L}$ as a parameter

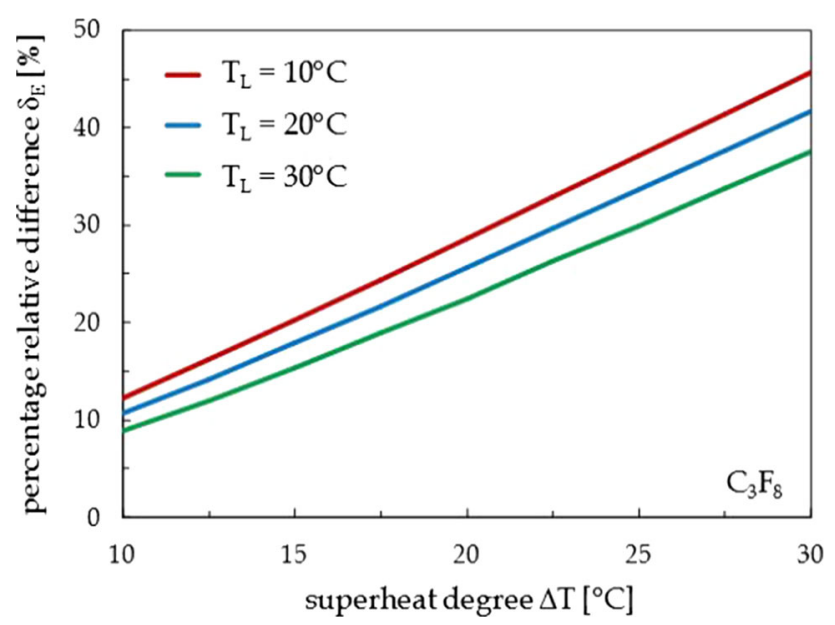

Fig. 7 Distributions of $\delta_{\mathrm{E}}=\left(\mathrm{E}_{\mathrm{c}}-\mathrm{E}_{\mathrm{c}}^{*}\right) / \mathrm{E}_{\mathrm{c}}$ vs. $\Delta \mathrm{T}$ for $\mathrm{C}_{3} \mathrm{~F}_{8}$ using $\mathrm{T}_{\mathrm{L}}$ as a parameter

between the two values becomes significantly high, almost regardless of the liquid temperature $T_{L}$.

All things considered, due to the higher critical energies obtained using (16) and (29), their application can be regarded as a prudential approach to the problem, resulting in what we could call an upper theoretical limit of the thermodynamic energy threshold. Of course, as long as only reversible processes are here accounted for, even higher energy thresholds can be detected experimentally, due to either dynamic losses and/or ineffective energy release by the stopping recoiled ion.

Finally, it seems interesting to examine the theoretical prediction of the combination of (16) and (29) using experimental data available in the literature. To this end, we chose an experimental result recently obtained for liquid Xenon, which seems to be more indicated than the other usual target liquids to test a novel critical energy theoretical equation.
In fact, at any recoil energy, an ion of Xenon travelling in pure liquid Xenon has a stopping force quite higher than, for example, that of ${ }^{12} \mathrm{C}$ or ${ }^{19} \mathrm{~F}$ in liquid $\mathrm{C}_{3} \mathrm{~F}_{8}$, which means that the additional condition for bubble nucleation requiring that the critical energy must be released inside a volume of characteristic dimension $R_{c}$ is more accurately satisfied. On the other hand, the use of a single-atom target eliminates the uncertainties on how to account for the relative contribution of the ions of a multi-atom molecule in determining the threshold.

The cited threshold measurement was performed by Baxter et al. [38] using a 30-g Xenon bubble chamber operated at 30 psia and $-60{ }^{\circ} \mathrm{C}$, whose corresponding critical energy calculated by the Bugg's equation in combination with (18) would be $8.3 \mathrm{keV}$. Indeed, the observed single and multiple bubble rates consequent to a $3.1 \mathrm{~h}$ exposure to $\mathrm{a}^{252} \mathrm{Cf}$ neutron source were consistent with the absolute rates predicted by a Monte Carlo simulation of the equipment executed using the MCNPX-POLIMI package assuming that the minimum nuclear recoil energy required to nucleate a vapour bubble was $19 \pm 6 \mathrm{keV}$ where, according to the authors, the range was dominated by the $30 \%$ uncertainty in their source strength. Conversely, the application of the relationships proposed for the calculation of $E_{c}$ and $R_{c}$, i.e., (16) and (29), results in a critical energy equal to $20.2 \mathrm{keV}$, with an uncertainty that could tentatively be assumed to be of the same order cited earlier.

Although we are aware that only reversible processes are considered in the calculation of the thermodynamic energy threshold, the difference between the measured value of $19 \pm 6 \mathrm{keV}$ and the predicted value of $8.3 \mathrm{keV}$ seems too high to be interpreted as the energy which goes into irreversible processes, even including the energy losses to scintillation. In fact, according to the measurements performed on liquid Xenon by different research teams - see for example Akerib et al. [39] - the scintillation yield for nuclear recoils between 1 and $100 \mathrm{keV}$ is widely lower than $15 \%$, which lies well inside the indicated $30 \%$ uncertainty range. Of course, the comparison with the Xenon result is not an evidence for our calculation, yet it represents a concordance with the earlier statement that our approach may be regarded as an upper theoretical limit of the thermodynamic energy threshold, encouraging enough to lead us to schedule future investigations on this same topic.

\section{Conclusions}

The relationships currently available for the calculation of the critical energy required for homogeneous nucleation in a superheated liquid, $\mathrm{E}_{\mathrm{c}}$, and the corresponding critical radius of the nucleated vapour bubble, $\mathrm{R}_{\mathrm{c}}$, show a number of inconsistencies, which has motivated the present study. Based on 
the procedure followed to obtain them, the pair of equations proposed here for the calculation of $E_{c}$ and $R_{c}$ turn out to be more consistent with the physical facts, the first being based on the application of the first law of thermodynamics, the second being derived under the assumption that the extreme instability condition represented by the criticallysized vapour bubble must correspond to a maximum of the difference between the free enthalpies of the metastable liquid and the stable vapour phases. An encouraging good agreement has been found between our theoretical prediction and an experimental result recently reported for Xenon at 30 psia and $-60{ }^{\circ} \mathrm{C}$. Further investigations on this topic are scheduled to be conducted in the next future.

Acknowledgements The authors are grateful to Donald Cundy for the valuable discussions and suggestions and for his help in reviewing the manuscript.

Data Availability Statement This manuscript has no associated data or the data will not be deposited. [Authors' comment: This is a theory paper so there is no data to show.]

Open Access This article is distributed under the terms of the Creative Commons Attribution 4.0 International License (http://creativecomm ons.org/licenses/by/4.0/), which permits unrestricted use, distribution, and reproduction in any medium, provided you give appropriate credit to the original author(s) and the source, provide a link to the Creative Commons license, and indicate if changes were made. Funded by SCOAP ${ }^{3}$.

\section{References}

1. D.A. Glaser, Phys. Rev. 87, 655 (1952)

2. W.J. Bolte et al., Nucl. Instrum. Methods Phys. Res. A 577, 569573 (2007)

3. E. Behnke et al., Phys. Rev. D 88, 021101 (2013)

4. M. Felizardo et al., E3S Web Conf. 12, 03002 (2016)

5. E. Behnke et al., Astropart. Phys. 90, 85-92 (2017)
6. C. Amole et al., Phys. Rev. Lett. 118, 251301 (2017)

7. The MOSCAB Collaboration, Eur. Phys. J. C 77, 752 (2017)

8. F. Seitz, Phys. Fluids 1, 2-13 (1958)

9. I.A. Pless, R.J. Plano, Rev. Sci. Instrum. 27, 935-937 (1956)

10. D.V. Bugg, Progr. Nucl. Phys. 7, 2-52 (1959)

11. A. Norman, P. Spiegler, Nucl. Sci. Eng. 16, 213-217 (1963)

12. A.G. Tenner, Nucl. Instrum. Methods 22, 1-42 (1963)

13. Ch. Peyrou, Bubble and Spark Chambers (Academic, New York, 1967)

14. C.R. Bell et al., Nucl. Sci. Eng. 53, 458-465 (1974)

15. H. Ing et al., Radiat. Meas. 27, 1-11 (1997)

16. P. Denzel et al., Phys. Rev. E 93(1), 013301 (2016). arXiv:1601.07390 [physics.comp-ph]

17. V.P. Skripov, Metastable Liquids (Wiley, New York, 1974)

18. L.W. Deitrich, T.J. Connolly, Nucl. Sci. Eng. 50, 273-282 (1973)

19. F. d'Errico, Radiat. Prot. Dosim. 84, 55-62 (1999)

20. S. Archambault et al., New J. Phys. 13, 043006 (2011)

21. C. Amole et al., Phys. Rev. Lett. 114, 231302 (2015)

22. B. DiGiovine et al., Nucl. Instrum. Methods Phys. Res. A 781, 96-104 (2015)

23. F.H. Poesposoetjipto, E. Hugentobler, Helv. Phys. Acta 43, $203-$ $222(1979)$

24. M.J. Harper, Nucl. Sci. Eng. 114, 118-123 (1993)

25. M.J. Harper, J.C. Rich, Nucl. Instrum. Methods Phys. Res. A 336 220-225 (1993)

26. M. Das, T. Sawamura, Nucl. Instrum. Methods Phys. Res. A 531, 577-584 (2004)

27. I. Lazanu, S. Lazanu, Roman. Rep. Phys. 63, 707-716 (2011)

28. F. d'Errico et al., Radiat. Prot. Dosim. 70(1997), 109-112 (1997)

29. S.C. Roy, Radiat. Phys. Chem. 61, 271-281 (2001)

30. S.C. Roy, B. Roy, Curr. Sci. 84, 516-528 (2003)

31. E. Fermi, Thermodynamics (Dover Publications Inc., New York, 1956)

32. L.D. Landau, E.M. Lifshitz, Statistical Physics (Pergamon Press Ltd., Oxford, 1959)

33. S. Auer, D. Frenkel, Nature 409(2001), 1020-1023 (2001)

34. S. Sandhya et al., Cryst. Res. Technol. 47, 780-792 (2012)

35. D. Yan et al., J. Phys. Chem. C 120, 10368-10380 (2016)

36. G. Du et al., J. Cryst. Growth 441(2016), 117-123 (2016)

37. P.J. Linstrom, W.G. Mallard (eds.), NIST Chemistry WebBook. https://doi.org/10.18434/T4D303.

38. D. Baxter et al., Phys. Rev. Lett. 118, 231301 (2017)

39. D.S. Akerib et al.,. arXiv:1608.05381v2 